\title{
Le rôle ambigu de la grande vitesse ferroviaire dans la gouvernance du corridor méditerranéen espagnol
}

The ambiguous role of high-speed rail in the Spanish Mediterranean railway corridor's governance

Die doppeldeutige Rolle der Hochgeschwindigkeitszug in der Governance von Mittelmeerseisenbahnkorridor in Spanien

Eloïse Libourel

\section{CpenEdition}

\section{Journals}

Electronic version

URL: http://journals.openedition.org/rge/5539

ISSN: 2108-6478

Publisher

Association des géographes de l'Est

Printed version

Date of publication: 1 October 2015

ISSN: 0035-3213

Electronic reference

Eloïse Libourel, « Le rôle ambigu de la grande vitesse ferroviaire dans la gouvernance du corridor méditerranéen espagnol », Revue Géographique de l'Est [Online], vol.55 / n³-4 | 2015, Online since 11 October 2015, connection on 08 September 2020. URL : http://journals.openedition.org/rge/5539

This text was automatically generated on 8 September 2020.

Tous droits réservés 


\section{Le rôle ambigu de la grande vitesse ferroviaire dans la gouvernance du corridor méditerranéen espagnol}

The ambiguous role of high-speed rail in the Spanish Mediterranean railway corridor's governance

Die doppeldeutige Rolle der Hochgeschwindigkeitszug in der Governance von Mittelmeerseisenbahnkorridor in Spanien

Eloïse Libourel

\section{Introduction}

1 Le corridor méditerranéen est un projet d'infrastructure ferroviaire exprimé à plusieurs niveaux qui ne se recouvrent que partiellement. Il s'agit d'abord de l'un des neuf «corridors multimodaux » formant le «réseau central » européen défini par la Commission européenne en 2013 (Commission européenne, 2013) au titre des RTE-T (Réseaux Transeuropéens de Transport), dans lesquels il est présent depuis l'origine (Commission européenne, 1996, 2005). Le corridor méditerranéen est également un projet espagnol inscrit dans les documents de planification successifs depuis les années 1990 (Ministerio de Fomento, 2005, 2012; MOPT, 1993), ainsi que dans les débats politiques à l'échelle nationale, régionale et locale. Enfin, c'est la dénomination choisie par le lobby économique Ferrmed, installé à Bruxelles et fondé par un Catalan, pour désigner sa proposition de grand axe de fret ferroviaire de Casablanca à SaintPétersbourg.

2 La mise en concordance de ces trois approches du corridor méditerranéen est problématique, car elle implique des visions très différentes du projet, tant du point de vue de l'infrastructure et de son affectation que du point de vue de l'aménagement des territoires traversés. De plus, de nombreux acteurs, à différents niveaux institutionnels, sont impliqués dans le projet de corridor méditerranéen, auquel ils 
apportent leurs référents identitaires et politiques propres, ainsi que leurs objectifs particuliers. L'Union européenne a un rôle de prescripteur et de financeur, mais c'est bien l'État espagnol qui a la compétence exclusive en matière d'infrastructures de transport nationales; les villes sont quant à elles compétences pour définir les projets urbains et notamment les projets de quartiers de gares, tandis que les Communautés autonomes n'ont théoriquement pas de pouvoir en matière de grande vitesse. À ces acteurs institutionnels, il convient d'ajouter également d'autres acteurs, publics ou privés, qui obéissent à leurs propres logiques, parmi lesquels les fédérations d'entreprises et le milieu universitaire sont particulièrement actifs. Si ces acteurs n'ont pas de compétence directe, ils s'organisent et ont une influence réelle sur les choix politiques.

3 Au sein des différents projets, la question de la grande vitesse n'est pas centrale; elle est néanmoins un enjeu majeur du corridor méditerranéen. En effet, si le fret ferroviaire en est l'élément principal, c'est bien la grande vitesse qui est au centre de tous les discours. Ce paradoxe apparent s'explique notamment par l'image positive a priori véhiculée par la grande vitesse : facteur de modernité pour les métropoles, elle est également plus facilement acceptée et comprise par les citoyens-électeurs. Elle devient dès lors un levier de la négociation et de la discussion élevée sur la place publique. En ce sens, la question de la grande vitesse fonctionne comme une plateforme pour l'expression des revendications des différents acteurs, et ce en dépit de son caractère secondaire dans le projet. Il est donc intéressant d'analyser la manière dont cette question participe de la recomposition du schéma de gouvernance théorique qu'elle soit gouvernance territoriale ou gouvernance de projet - du corridor méditerranéen. Aujourd'hui, le tracé d'un corridor méditerranéen à grande vitesse de la frontière française à Algésiras est présent dans le plan d'infrastructures espagnol en vigueur. La partie comprise entre la frontière et Valence est également incluse dans les RTE-T de 2012. Toutefois, la mise en œuvre du projet est à l'arrêt.

Cette analyse s'appuie sur un important travail de documentation qui prend en compte l'ensemble des plans d'infrastructures espagnols (Ministerio de Fomento, 2005, 2012 ; MOPT, 1993) et de la programmation européenne (Commission européenne, 1996, 2005, 2013) concernant le corridor méditerranéen, ainsi que les propositions publiées par les lobbies (CENIT, 2012 ; Ferrmed, 2009) ou à l'initiative d'acteurs locaux, publics ou privés (Camisón Zozorna, 2012 ; Institut Ignasi Villalonga, 2010). L'identification fine de ces acteurs a constitué un préalable essentiel permettant d'analyser et de confronter leurs discours à travers les médias, les conférences thématiques que certains d'entre eux organisent et une série d'une cinquantaine d'entretiens réalisés sur place entre 2011 et 2014. Dans ce corpus relativement vaste, l'élément le plus problématique est de distinguer ce qui relève spécifiquement de la grande vitesse dans des discours fournis mais adoptant souvent une définition floue du corridor méditerranéen.

5 La première partie de cet article présente le double contexte de gouvernance espagnol et européen qui structure le débat sur le corridor méditerranéen, et pose la question de l'émergence d'un modèle singulier de gestion du processus de décision. Nous proposons ensuite une analyse détaillée de la place de la grande vitesse ferroviaire dans les discours des différents acteurs concernés, à toutes les échelles, par ce corridor. Enfin, la dernière partie est consacrée à la question de la territorialisation du corridor méditerranéen à travers les changements de gouvernance induits par la grande vitesse. 


\section{Enjeux et acteurs du corridor méditerranéen au prisme de son double contexte espagnol et européen}

6 L'inscription concomitante du corridor méditerranéen dans les documents-cadres de programmation de la Commission européenne et dans les plans d'infrastructures du gouvernement espagnol n'est pas la simple traduction au niveau national d'un principe adopté au niveau européen. En effet, l'axe méditerranéen est stratégique pour les régions littorales et la revendication d'un réseau ferroviaire qui les relierait à l'Europe existe depuis les années 1920 (Abad García, 1981), posant à Madrid la question de sa centralité et de sa prépondérance dans le réseau ferroviaire. Ce corridor, revendiqué de longue date mais jamais pleinement réalisé, est désormais officiellement une priorité européenne, ce qui ajoute une strate institutionnelle et politique supplémentaire à la question.

\section{A. La gouvernance au(x) défi(s) du corridor méditerranéen}

7 Le débat sur le projet de corridor méditerranéen fait émerger trois enjeux majeurs en matière de gouvernance: le passage d'un système traditionnel de gouvernement en matière d'infrastructures à un processus complexe de gouvernance à différentes échelles, la prise en compte du corridor comme un objet complexe échappant aux logiques habituelles de l'aménagement et de la gouvernance territoriale, et l'ambiguïté de la nature même du corridor méditerranéen. En effet, la place de la grande vitesse ferroviaire dans le projet n'étant pas clairement définie, les rapports de force entre acteurs et les enjeux qui touchent les différentes échelles territoriales restent mouvants.

8 Le projet de corridor méditerranéen interroge les processus habituels de gouvernance en matière d'infrastructures. En effet, la planification et la réalisation des grandes infrastructures de transport relève en Espagne de la compétence de l'État. Les décisions en la matière sont prises à Madrid et s'imposent aux Communautés autonomes. On est donc théoriquement dans un système de gouvernement. De plus en plus souvent, une double négociation a lieu en amont, avec l'Union européenne - financeur et de plus en plus souvent prescripteur à travers les RTE-T - d'une part, et avec les régions - dont les revendications dans le domaine ferroviaire appuient les revendications autonomiques d'autre part, ce qui fait évoluer le processus de prise de décision vers une forme de gouvernance de projet dans laquelle les acteurs institutionnels ont la part belle, mais qui intègre aussi les acteurs économiques (via les Chambres de Commerce et d'Industrie et les associations d'entreprises notamment) et universitaires. De plus, le cas du corridor méditerranéen est d'autant plus complexe que son statut de projet européen l'inscrit dans le cadre communautaire du principe de subsidiarité : les régions sont directement représentées à Bruxelles (Rey-Valette et al., 2014), et les instances européennes traitent de plus en plus volontiers avec cet échelon. L'introduction du corridor méditerranéen dans la planification européenne tend à brouiller la lisibilité des compétences de chaque acteur. Le processus de gouvernance qui se met en place autour du corridor méditerranéen s'inscrit donc dans une logique multiscalaire complexe.

9 En outre, la notion-même de corridor est complexe et donne corps à un objet aux dimensions mal définies. En effet, si la notion a d'abord été employée en économie, puis 
pour la description des systèmes de villes (Debrie et Comtois, 2010), elle désigne désormais dans le cadre européen de grands axes transcontinentaux multimodaux dont la réalité infrastructurelle reste à construire. L'extension et les caractéristiques techniques des corridors sont des points en cours de définition, si bien que certains auteurs préfèrent parler d'un "faisceau d'infrastructures" (Priemus et Zonneveld, 2003, p. 167). Pour ces auteurs, "le développement de corridors concerne les connexions qui utilisent des modes de transport différents (par exemple la voiture, le train, le tramway, le bateau, l'avion) et transportent à la fois des passagers et du fret ». Le corridor méditerranéen pose en l'espèce un double défi dans sa longueur et dans sa largeur. En effet, son extension soulève des problématiques spécifiques car il est transfrontalier, transrégional et interopérable, autant de caractéristiques dont la gestion complexe s'ajoute à celle de la construction d'un nouvel axe. Dans sa largeur, le corridor - en tant qu'ensemble d'infrastructures et de territoires - pose également problème car ses limites spatiales sont floues: il est possible de considérer une définition plus ou moins large, incluant dans le corridor les villes, les populations et les activités qui lui sont liées, comme le suggèrent les définitions les plus souvent admises (Prentice, 1996; Whebell, 1969), mais se pose alors la question de la définition de l'aire d'influence du corridor méditerranéen. Dès lors que l'on considère cet objet dans sa longueur et sa largeur, le nombre et la nature des acteurs concernés s'accroît, complexifiant d'autant la mise en place d'une gouvernance.

Enfin, la place ambiguë de la grande vitesse dans le corridor méditerranéen contribue à la complexité du processus de prise de décision en donnant à la gouvernance du projet un double visage. Parmi les acteurs qui s'investissent dans les débats, certains sont concernés prioritairement par le fret et d'autres par les voyageurs. Cette situation liée à l'indéfinition entretenue dans les plans nationaux d'infrastructures successifs, conduit à brouiller les débats, certains acteurs n'hésitant pas à jouer sur le double tableau, quitte à distordre leur discours en s'appuyant sur l'aspect qui offre la meilleure image de marque - la grande vitesse - pour favoriser un projet qui doit avant tout répondre à leurs objectifs de transport de fret. Aussi la plupart des personnalités politiques régionales catalanes et valenciennes font-elles une publicité importante pour la desserte en AVE (grande vitesse espagnole) auprès de leurs électeurs mais soutiennent-elles fortement, quoique de manière parfois moins ostensible, le corridor de fret, principale revendication du secteur des entreprises et des ports.

\section{B. De multiples acteurs pour un processus de gouvernance complexe}

11 Dans ce contexte où la place de la grande vitesse est à la fois centrale et mal assurée, le jeu des acteurs, quoique difficilement saisissable, est essentiel. Il est d'autant plus complexe que les incertitudes maintenues laissent une porte ouverte à toutes les interprétations et revendications.

Les acteurs concernés par le corridor méditerranéen peuvent être classés en plusieurs groupes en fonction de l'échelle territoriale à laquelle ils agissent d'une part et en fonction de leur nature - publique ou privée - d'autre part. Toutefois, l'échelle principale d'action, envisagée au prisme du corridor méditerranéen, ne coïncide que rarement avec l'échelle à laquelle s'expriment les revendications. Les entreprises du secteur industriel, quoique représentantes de clusters d'activités ancrés dans un 
territoire régional (ainsi l'industrie agro-alimentaire de Murcie), élèvent leurs revendications à une échelle continentale qui est celle de leur marché potentiel. D'une manière encore plus marquante, les élus des villes littorales qui cherchent dans la grande vitesse ferroviaire un facteur de développement urbain mettent en avant la quête d'un statut métropolitain à l'échelle de l'Europe ou du Bassin Méditerranéen. La simple classification par les échelles est donc ici inopérante.

La typologie des acteurs par leur champ d'appartenance principal - acteurs politiques, économiques ou de la société civile - permet de mettre en avant l'interaction de ces différents champs. Mais là aussi, la classification est inopérante, car de nombreuses personnalités du monde universitaire par exemple sont également étroitement liées à la vie politique ou à la vie économique, voire aux deux. En effet, Josep Vicent Boira i Maiques, professeur de géographie à l'Université de Valence, participe étroitement aux activités de l'Institut Ignasi Villalonga qui porte le valencianisme politique (Fabra, 1975) et regroupe les intérêts économiques de la Communauté autonome pour revendiquer un axe euro-méditerranéen (Institut Ignasi Villalonga, 2010). De même, le professeur d'économie de l'Université de Valence César Camisón Zozorna dirige les études commanditées par l'Association valencienne des entrepreneurs - dont l'action de lobbying est réelle - sur le corridor méditerranéen. Les cercles se recoupent donc. Il est toutefois possible d'identifier, à travers les entretiens menés avec les principaux acteurs et à travers leurs discours exprimés dans les media et à l'occasion de conférences sur le corridor méditerranéen, l'interaction de ces différents champs.

Tableau 1 : Pluralité des acteurs, pluralité de la place de la grande vitesse dans le corridor méditerranéen

\begin{tabular}{|c|c|c|c|c|}
\hline Acteur & Echelle d'appartenance & Echelle d'action & Objectifs principaux & $\begin{array}{l}\text { Prise en compte de la } \\
\text { grande vitesse }\end{array}$ \\
\hline Commission européenne & Européenne & Européenne et régionale & $\begin{array}{l}\text { Promouvoir l'interopérabilité des } \\
\text { réseaux }\end{array}$ & $\begin{array}{l}\text { OUI : comme une des } \\
\text { modalités possibles du } \\
\text { corridor }\end{array}$ \\
\hline Gouvernement espagnol & Nationale & Nationale et européenne & $\begin{array}{l}\text { Compléter le réseau de } \\
\text { passagers et garantir l'inscription } \\
\text { d'un projet RTE-T }\end{array}$ & $\begin{array}{l}\text { OUI : dans une perspective } \\
\text { radiale }\end{array}$ \\
\hline Groupe Fomento (ADIF + Renfe) & Nationale & Nationale & $\begin{array}{l}\text { Améliorer le réseau national, } \\
\text { mettre les grands axes aux } \\
\text { normes européennes }\end{array}$ & $\begin{array}{l}\text { OUI : dans une perspective } \\
\text { radiale }\end{array}$ \\
\hline $\begin{array}{l}\text { Communautés autonomes } \\
\text { méditerranéennes }\end{array}$ & Régionale & Européenne & $\begin{array}{l}\text { Intégration euro-régionale, } \\
\text { connexion des ports, axe littoral }\end{array}$ & OUI : comme axe littoral \\
\hline$\overline{\text { Villes }}$ & Locale & Locale et européenne & $\begin{array}{l}\text { S'insérer dans le concert } \\
\text { métropolitain euro- } \\
\text { méditerranéen }\end{array}$ & $\begin{array}{l}\text { OUI : comme facteur de } \\
\text { développement et de } \\
\text { rayonnement }\end{array}$ \\
\hline Plate-forme $E U$ Core Net Cities & Européenne & Européenne et urbaine & $\begin{array}{l}\text { Rassembler les villes nodales du } \\
\text { "réseau de base" européen }\end{array}$ & NON \\
\hline $\begin{array}{l}\text { Lobbies (Ferrmed, Business } \\
\text { Eumed) }\end{array}$ & Européenne & Européenne et locale & $\begin{array}{l}\text { Connecter le littoral } \\
\text { méditerranéen à l'Europe, } \\
\text { rassembler les entreprises } \\
\text { méditerranéennes }\end{array}$ & NON \\
\hline Autorités portuaires & Locale ou régionale & Nationale et européemne & $\begin{array}{l}\text { Améliorer leur connexion au } \\
\text { marché européen }\end{array}$ & NON \\
\hline $\begin{array}{l}\text { Entreprises (CCI, Associations } \\
\text { d'entreprises...) }\end{array}$ & Locale ou régionale & Locale et macro-régionale & $\begin{array}{l}\text { Connecter le littoral } \\
\text { méditerranéen à l'Europe, } \\
\text { rassembler les entreprises } \\
\text { méditerranéennes }\end{array}$ & $\begin{array}{l}\text { PEU : comme un atout } \\
\text { secondaire pour le littoral }\end{array}$ \\
\hline Universitaires & Locale & $\begin{array}{l}\text { Régionale et nationale } \\
\text {. }\end{array}$ & $\begin{array}{l}\text { Promouvoir une macro-région } \\
\text { méditerranéenne, renforcer le } \\
\text { poids économique de la } \\
\text { Méditerranée en Europe }\end{array}$ & $\begin{array}{l}\text { PEU : comme un atout } \\
\text { secondaire, voire comme } \\
\text { une opération peu rentable }\end{array}$ \\
\hline Instituts (IIVEE- Euram...) & Locale et régionale & $\begin{array}{l}\text { Régionale } \\
\text { Ren }\end{array}$ & $\begin{array}{l}\text { Combiner les différents acteurs } \\
\text { autour d'un projet méditerranéen }\end{array}$ & $\begin{array}{l}\text { PEU : comme un atout } \\
\text { secondaire pour le littoral }\end{array}$ \\
\hline
\end{tabular}

En reprenant une classification par échelles et en la mettant en perspective avec le type d'acteurs et leurs objectifs annoncés, l'on obtient une vision un peu plus claire de la situation. Ce classement est issu d'un travail d'entretiens qui a permis de définir les objectifs de chacun des acteurs de manière fine. Souvent des ambiguïtés existent dans 
les intérêts des uns et des autres. En ce qui concerne la grande vitesse, il ressort qu'elle est importante pour tous les échelons d'actions, mais à des titres divers selon les acteurs (voir Tableau 1). En effet, certains ne plaident qu'en faveur d'un corridor de fret, jugé plus important pour l'activité économique des régions méditerranéennes, mais cela ne les empêche pas de s'emparer à l'occasion de la question des passagers, avec des objectifs politiques. C'est ainsi qu'un groupe formé d'entreprises participant à l'Association valencienne des entrepreneurs - qui défend un corridor de fret - dont le sigle, AVE, rappelle toutefois le nom de la grande vitesse espagnole, s'engage en faveur de la grande vitesse sous le nom de Pro-AVE.

Si ces propositions sont complémentaires, chacune présente des limites liées au caractère très singulier du jeu de gouvernance du corridor méditerranéen. En effet, la place ambiguë de la grande vitesse contribue à complexifier le jeu des acteurs. Cette thématique, bien plus que celle du fret, appelle des réalisations marquantes à l'échelle locale, où l'aménagement de gares et le renouvellement urbain associé sont, plus que des infrastructures liées au projet de corridor méditerranéen, des arguments politiques.

16 C'est pourquoi l'on se trouve, dans le cas précis du corridor méditerranéen, à la frontière entre la gouvernance territoriale et la gouvernance de projet. En effet, la multiplication des échelons dans la prise de décision est devenue une caractéristique majeure des projets européens, soumis à l'approbation des États, mais également des échelons inférieurs, en vertu du principe de subsidiarité, avec une participation de plus en plus grande des acteurs non-institutionnels (Chevallier, 2003 ; Romero González, 2012). Cette implication croissante de l'Union européenne dans l'aménagement du territoire affecte également la gouvernance territoriale proprement dite (Bonerandi et Santamaria, 2011; Romero González, 2005). En effet, le choix d'implantation de nouveaux équipements et les choix territoriaux sont discutés à différentes échelles et souvent dans des processus de décision participatifs. À titre d'exemple, le projet Valencia Parque Central de gare souterraine recouverte d'un grand parc urbain de plus de 20 hectares et accompagnée d'un programme de renouvellement urbain visant à créer une nouvelle centralité à Valence a été conçu en partenariat avec la Communauté autonome et l'État, et il a été soumis à une consultation publique (Libourel, 2011). Ce projet est mené par une société d'économie mixte regroupant les acteurs étatiques (Ministère des Transports, ADIF et Renfe), la Communauté autonome et la Ville de Valence, et il s'appuie, comme à Barcelone, sur un modèle de financement reposant sur les investisseurs privés : l'investissement public initial doit être compensé par la vente des terrains à bâtir dégagés au-dessus de la gare. C'est bien le thème de la grande vitesse qui permet le passage d'une gouvernance de projet à une gouvernance territoriale car c'est lui qui a les implications les plus directement perceptibles dans le territoire à l'échelle locale.

17 Ainsi, le cas du corridor méditerranéen, par sa double orientation vers le fret et vers la grande vitesse, et parce qu'il est à la croisée d'un système contesté de gouvernement national (qui possède la compétence exclusive d'aménagement des grandes infrastructures de transport) et d'une gouvernance européenne complexe, semble tendre vers l'établissement d'un processus spécifique de "gouvernance de corridor ». En ce sens, la nature même de l'objet étudié dans sa complexité géographique et institutionnelle appelle une redéfinition de la gouvernance territoriale européenne. 


\section{La grande vitesse dans le corridor méditerranéen : une vision au centre de la gouvernance territoriale}

18 La grande vitesse ferroviaire, partie du projet de corridor méditerranéen, a un rôle majeur dans la redéfinition de la gouvernance territoriale parce qu'elle apparaît comme l'enjeu le plus visible, dégageant l'image de marque la plus positive et mobilisant le plus largement les acteurs locaux. Cette place centrale de la grande vitesse dans les processus de prise de décision, contrastant avec le caractère marginal $\mathrm{du}$ transport de passagers dans le projet européen comme dans les derniers développements des documents de programmation espagnols (les restrictions budgétaires consécutives à la crise ont amené à une réduction de la voilure du réseau AVE projeté), est liée à l'investissement symbolique qu'elle revêt pour les différents acteurs.

\section{A. Des visions différentes de la grande vitesse selon les acteurs}

19 La charge symbolique dont la grande vitesse est lestée par les différents acteurs du corridor méditerranéen transparaît dans leurs discours. En effet, qu'ils soient publics ou privés, institutionnels ou non, la plupart des acteurs du corridor méditerranéen sont concernés par la question. Tous n'envisagent pas le transport de passagers comme un enjeu central (voir Tableau 1), mais conscients que le thème est plus porteur que celui $\mathrm{du}$ fret dans l'opinion publique, ils lui marquent leur soutien à des degrés divers. Le train à grande vitesse est perçu comme une opportunité non seulement économique mais aussi politique.

Les acteurs nationaux et régionaux s'emparent de la question de la grande vitesse ferroviaire au prisme de la construction du réseau et de son interconnexion avec le réseau français. Le choix fait à la fin des années 1980 par le gouvernement socialiste de González (en Espagne, les grandes infrastructures de transport sont la compétence exclusive du gouvernement central) de construire de toutes pièces un nouveau réseau aux normes européennes ouvrait la possibilité de changer la logique radiale qui avait jusqu'alors prévalu en Espagne et qui avait abouti au réseau ferroviaire et autoroutier en étoile autour de Madrid que l'on connaît aujourd'hui. Cette perspective de changement des priorités s'est un temps concrétisée dans la mise en service de l'Euromed, service reliant Barcelone à Alicante à vitesse élevée (et non aux standards de la grande vitesse) sur des voies conventionnelles. Toutefois, les LGV construites en Espagne depuis 1992 reproduisent le schéma radial d'aménagement du territoire, reliant Madrid à Séville, puis à Barcelone, à Valence et à Alicante. Pour les gouvernements successifs, l'enjeu de la grande vitesse est d'amarrer les capitales provinciales à la capitale nationale, renforçant le poids de cette dernière. Dans ce contexte, les Communautés autonomes littorales, au centre de la revendication d'un corridor méditerranéen, se mobilisent politiquement pour obtenir leur ligne à grande vitesse littorale qui désengorgerait une ligne conventionnelle saturée. Sous la pression politique de la Catalogne, le tracé de la ligne Madrid-Barcelone passe donc par Tarragone, ce qui la détourne du trajet le plus bref. La Communauté valencienne a également obtenu que l'extension de la LGV Madrid-Valence jusqu'à Castellón soit incluse dans les projets (elle n'est cependant pas réalisée). Ces pressions s'appuient sur le constat d'une saturation de l'axe méditerranéen, sur l'idée d'une continuité avec les 
réseau français, ainsi que sur la contestation des projets de LGV reliant Madrid à la Galice ou à l'Estrémadure, jugés non rentables économiquement et socialement, donc trop coûteux pour les finances de l'État.

21 Dans le contexte actuel de crise économique que traverse l'Espagne, le développement des lignes à grande vitesse, en particulier sur l'axe méditerranéen, est perçu par les acteurs locaux comme une opportunité de sortie de crise. Quoiqu'il puisse paraître paradoxal de miser sur d'importants efforts de construction d'infrastructures, de gares et de quartiers de gares pour résoudre une situation héritée de l'éclatement de la bulle immobilière, c'est bien le pari qui est pris par de nombreuses villes, au premier rang desquelles Barcelone et Valence. En effet, à l'échelle locale, la grande vitesse, financée en partie par l'État central avec d'importantes aides européennes au titre du corridor méditerranéen, représente une possibilité de relance de l'activité touristique et des affaires - ou du moins est-ce l'idée qui transparaît dans les entretiens menés auprès des services d'urbanisme et des sociétés d'économie mixte chargées de la mise en œuvre des projets. Sans que cela soit clairement exprimé la plupart du temps, l'idée est de profiter des financements nationaux et européens associés au corridor méditerranéen pour réaliser des projets d'échelle urbaine (Libourel, 2011).

Les acteurs urbains - municipalités, agences d'urbanisme et secteur touristique - sont les premiers à appuyer la grande vitesse au nom de la visibilité, de l'image et du rayonnement censés conférer à la ville une aura de métropole (Romero González et Farinós Dasí, 2012). Les programmes de coopération régionale ou internationale tendent souvent à définir les villes méditerranéennes par leurs lacunes en matière d'infrastructures, par opposition tacite aux villes du nord de l'Europe, perçues comme modèles (MEDOCC, 2004). L'acquisition d'une desserte par la grande vitesse et la construction à cette occasion d'une nouvelle gare moderne éventuellement support d'une vaste opération de renouvellement urbain est donc une double opportunité d'atteindre un statut métropolitain en Méditerranée et de se classer au rang des grandes villes modernes européennes.

La question des gares est en cela absolument centrale. De nombreux travaux de chercheurs espagnols font état des politiques d'insertion urbaine de la grande vitesse (Bellet, Alonso Logroño et Casellas, 2010 ; Bellet et Gutiérrez, 2011; Diaz Marquez et Ureña Francés, 2010 ; Santos y Ganges, 2007). En effet, si les villes ne possèdent pas de compétence en matière de grande vitesse, elles ont la possibilité de modifier les plans d'urbanisme en fonction des projets qui leur paraissent prioritaires: les projets de nouvelles gares ou de rénovation des anciennes gares, parce qu'ils impliquent une action urbanistique plus large concernant tout un quartier, sont des leviers politiques pour obtenir le tracé favorable d'une ligne. Au regard des différentes études de cas, il apparaît que la fréquence avec laquelle des projets de renouvellement urbain sont associés à l'arrivée de l'AVE est plus importante qu'ailleurs en Europe. Ceci est en partie lié à l'obligation d'adapter les gares aux nouvelles voies, la grande vitesse étant conforme aux standards européens de sécurité et d'écartement (1 $435 \mathrm{~mm}$ contre 1668 $\mathrm{mm}$ pour le réseau conventionnel de la Péninsule ibérique). Toutefois, c'est plutôt une volonté politique de rayonnement qui est à l'origine de ces nombreux projets, allant du quartier de gare à la mise en souterrain des infrastructures (voir Fig. 1). 
Fig. 1 : Projets de gares à grande vitesse associés à des opérations de renouvellement urbain
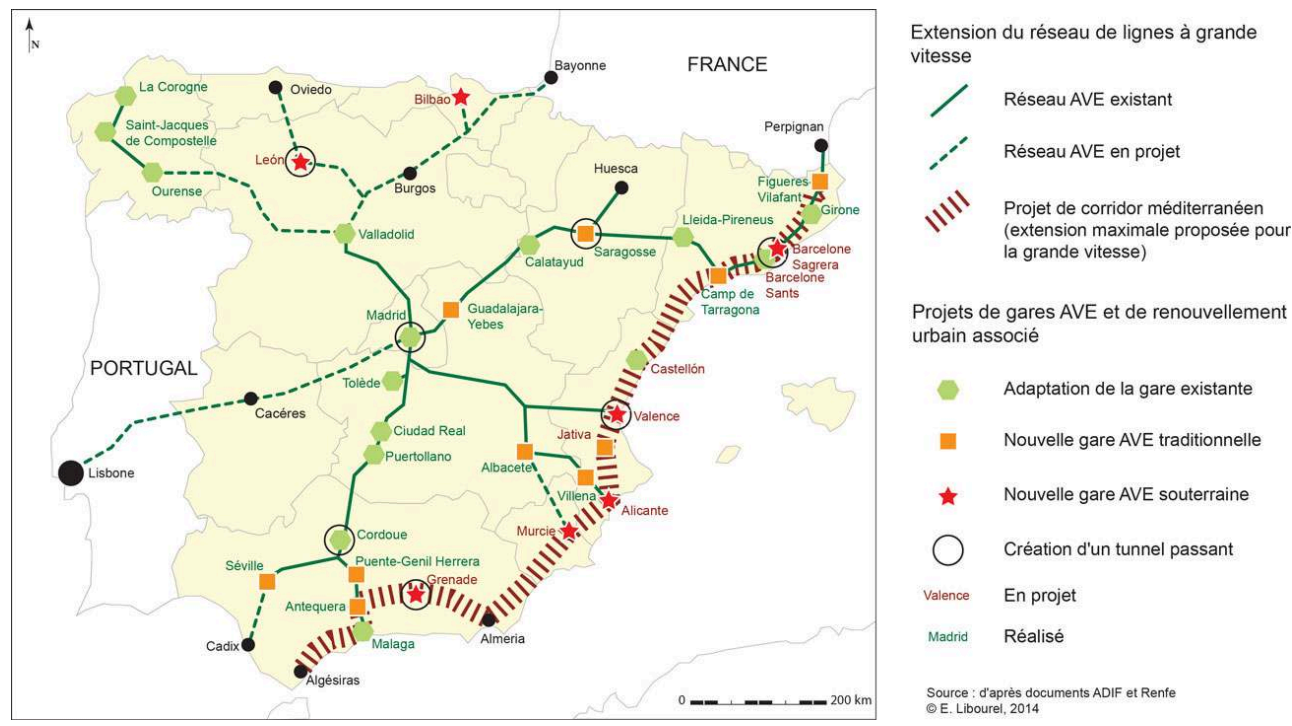

À titre d'exemple, la gare AVE de Saragosse a été construite sur la LGV MadridBarcelone à l'écart de la gare historique. Toutefois, c'est par elle que passent désormais également les trains régionaux. Le nouveau site et l'imposant bâtiment qui a été construit se situe entre la ville et le site de l'Exposition internationale. L'objectif d'en faire un train d'union urbain n'est toutefois pas réalisé car les accès à la gare sont malaisés pour les piétons et car la coulée verte initialement prévue n'a pas été aménagée. Nonobstant, la gare, résolument moderne, est un symbole pour la ville. La grande vitesse apparaît donc comme un puissant fédérateur local: intérêts économiques, politiques et urbains s'y rencontrent. En effet, au-delà des acteurs locaux, les entreprises privées soutiennent au moins tacitement, et parfois activement, la grande vitesse comme facteur d'attractivité régionale, comme aménité susceptible d'intéresser leur activité, mais aussi comme pourvoyeuse de nouveaux chantiers.

\section{B. La grande vitesse, au centre des rapports de forces multiscalaires}

Le passage d'une logique de gouvernement hiérarchisée (top-down) à une logique de gouvernance où les lobbies formés d'institutions locales et régionales et d'acteurs privés agissent à l'échelon européen (bottom-up) s'apparente à une inversion des logiques traditionnelles des politiques espagnoles d'aménagement. Dans le même temps s'opère un saut scalaire important des échelles locale et régionale jusqu'à l'échelle européenne, laissant de côté l'échelle nationale, celle du gouvernement central espagnol.

Cette situation fait écho aux relations tendues qui existent entre Madrid et les gouvernements autonomiques qui revendiquent un accroissement de leur pouvoir dans le domaine des infrastructures et une autonomie politique plus complète. Cette dernière passe notamment par une représentation directe à Bruxelles, non seulement au Comité des Régions, mais également par le biais de délégations ou de représentations permanentes. La Catalogne est particulièrement active en la matière (Trépier, 2013). Non seulement les élus manifestent avec vigueur leurs désaccords avec Madrid et leur revendication d'un corridor ne passant pas par la capitale, mais les principaux lobbies soutenant le projet ont été fondés par des Catalans, comme Ferrmed 
en 2004 à l'initiative de Joan Amorós. Business Eumed, groupement d'intérêts moins connu défendant l'implantation d'entreprises dans le Bassin méditerranéen et proposant l'idée d'un "train orbital méditerranéen", est dirigé par Rafael Puig et compte aussi Joan Amorós dans son bureau. Enfin, EU Core Net Cities, qui regroupe des villes situées sur le réseau central européen, a été fondé en 2013 à Barcelone et son secrétariat est assuré par Ferrmed. Un petit nombre de personnalités fortes structurent ainsi la partie la plus active des revendications.

Les illustrations de ces rapports de force et de l'implication de nombreux acteurs à toutes les échelles et à tous les niveaux ne manquent pas. Lors de l'inauguration de l'arrivée à Valence de l'AVE en octobre 2010, la photographie officielle montre des représentants de l'ensemble des échelons territoriaux : le Roi Juan Carlos et la Reine Sofía sont présents, le Président du gouvernement José Luís Zapatero, le Ministre des Transports José Blanco, le Président de la Communauté autonome de Valence Francisco Camps et la Maire de Valence Rita Barberá sont également là. En permettant des inaugurations médiatisées, la grande vitesse est un enjeu politique pour tous les échelons. Positive, on l'a vu, à l'échelle locale, l'arrivée de l'AVE dans les villes méditerranéennes permet certes de renforcer le poids des régions littorales, mais également, si l'on veut en faire une lecture inverse, de conforter Madrid dans sa situation dominante puisqu'aucune liaison transversale n'a encore été inaugurée. En outre, il est possible d'interpréter la diligence de gouvernement à doter Barcelone d'une part et Valence d'autre part de leur connexion à grande vitesse avec la capitale comme une manière d'ajourner la liaison entre les deux villes catalanophones.

La grande vitesse, quoique secondaire en termes de priorités d'aménagement, apparaitt donc néanmoins comme un objet central dans les rapports de forces entre les différents échelons institutionnels. Si elle est un facteur relativement consensuel entre les acteurs privés et publics, mais également entre partis politiques, comme cela a pu être le cas au Pays Basque (Audikana, 2012), l'unanimité de façade laisse la place à des tensions importantes entre échelons institutionnels. Loin de n'être qu'un enjeu secondaire, il semble que la grande vitesse comme objet approprié par tous les acteurs soit le point névralgique de cristallisation des enjeux de gouvernance du projet de corridor méditerranéen.

\section{La grande vitesse comme acteur de la gouvernance? Territorialiser le corridor méditerranéen}

La prise en compte des ambiguïtés du jeu d'acteurs autour de la grande vitesse dans le corridor méditerranéen invite à poser la question de la territorialisation, au sens anglosaxon du terme comme processus territorialisé qui permet de " comprendre comment un jeu d'acteurs se constitue en un lieu donné et le fait entrer dans une logique de projet» (Woessner, 2010, p. 671). Cette logique de projet suppose notamment une volonté de coopération entre les acteurs (Debarbieux et al., 2002; Vanier, 2009). Or l'axe méditerranéen est en quête d'une construction territoriale autour du corridor. La grande vitesse, parce qu'elle relierait les villes entre elles en peu de temps et avec un confort supérieur, pourrait en être le vecteur privilégié. 


\section{A. La grande vitesse, acteur du territoire}

31 La grande vitesse bénéficie, on l'a vu, d'une image de marque très positive. S'il est démontré que les «effets structurants» du transport sur le territoire relèvent essentiellement du mythe politique (Offner, 1993), ce mythe est encore souvent repris et relayé par les acteurs publics, soucieux de tirer parti auprès de leur électorat des projets de lignes à grande vitesse et de leurs effets supposés sur le développement local.

32 Ainsi, non seulement les inaugurations de gares et de nouvelles lignes attirent l'ensemble de la classe politique, mais les nouveaux équipements servent de support pour une communication autour de l'image de marque de la grande vitesse. Lors de l'arrivée de l'AVE à Valence, on a ainsi vu plusieurs acteurs s'approprier l'espace de la nouvelle gare Joaquín Sorolla, pourtant provisoire, à travers des supports visuels. Renfe, l'opérateur du réseau, déploie ainsi une campagne publicitaire jouant sur l'image du « cheval gagnant», avec un Talgo 350 (le train à grande vitesse espagnol) en fond sur un profil découpé de cheval fougueux.

Photo 1 : Publicité de Renfe dans la gare de Valence Joaquín Sorolla

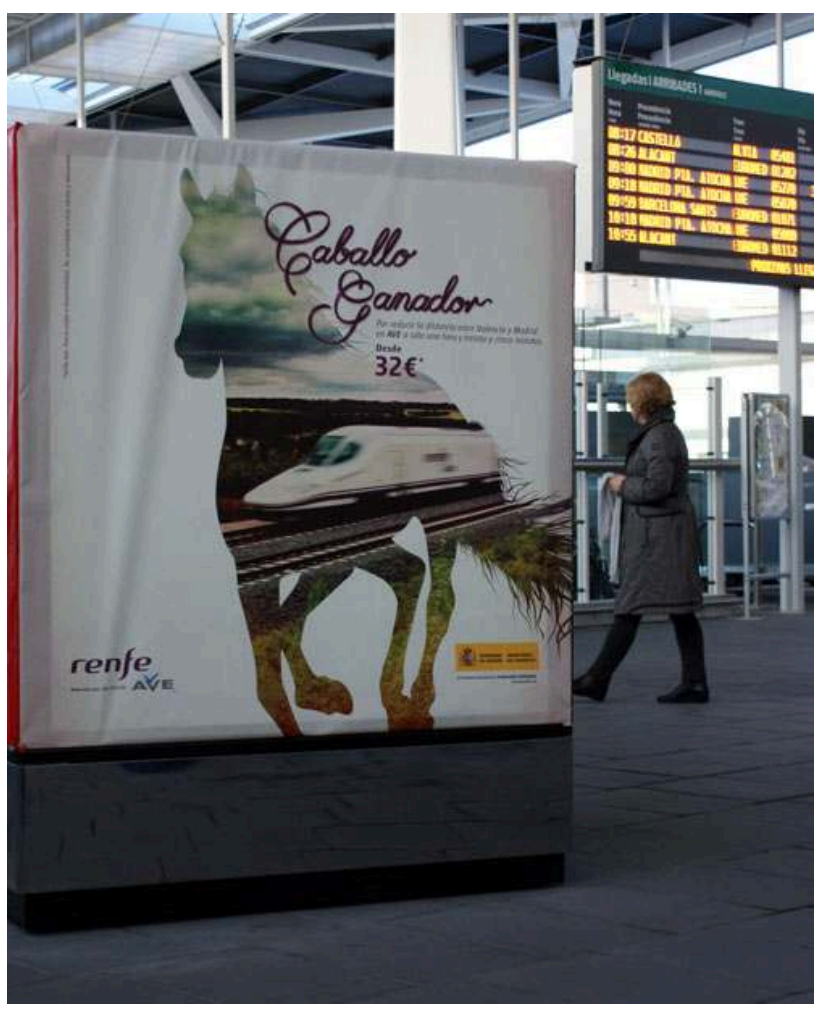

(C) E. Libourel, mars 2011

33 Au même moment, il y avait également en plusieurs endroits de la gare des dispositifs rappelant la distance à Madrid et le temps de trajet nécessaire en gros caractères et en trois dimensions, tels des totems. Ils étaient posés par ADIF, gestionnaire de l'infrastructure et des gares. 
Photo 2 : Dispositif mettant en valeur la distance Madrid-Valence dans la gare Joaquín Sorolla

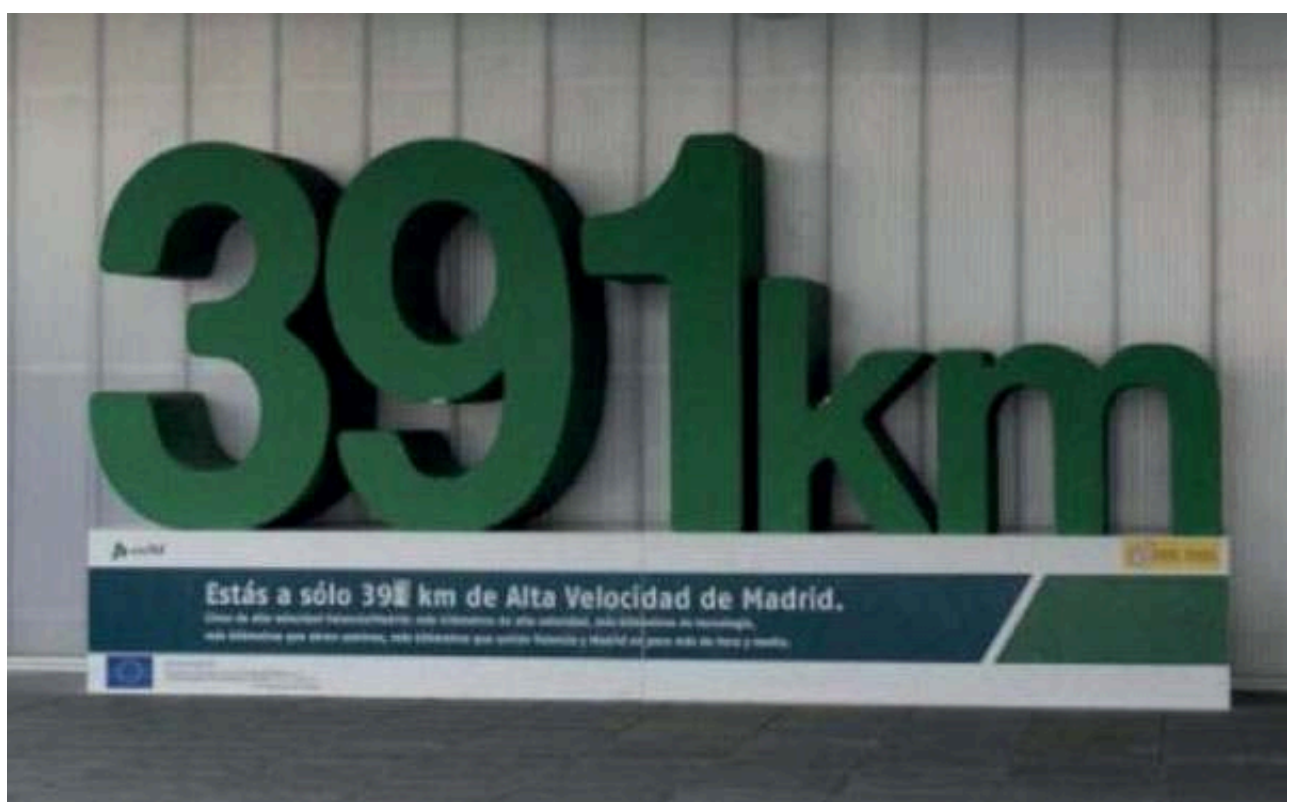

(c) E. Libourel, mars 2011 du projet éponyme, pour donner à voir et expliquer le projet de future gare souterraine et de parc urbain renouvelant le quartier. On voit par-là combien la grande vitesse est mise en valeur comme acteur de l'aménagement. C'est elle qui compresse les distances, qui dynamise le territoire et qui permet le renouvellement urbain. Elle est, à ce titre, un support pour une communication qui dépasse le strict cadre ferroviaire.

En effet, dans les années 2010, au moment où Valence est connectée à Madrid, alors que la grande vitesse dans le corridor méditerranéen, quoique présente dans les documents de planification (Ministerio de Fomento, 2005), est soumise aux incertitudes de financement de la crise, certaines villes moyennes et stations balnéaires, pour lesquelles l'enjeu est d'obtenir une gare, commencent à utiliser l'image de l'AVE. La ville de Gandía, en coopération avec les principaux acteurs du tourisme (fédérations hôtelières et agences de voyages essentiellement), lance dès 2010 une campagne publicitaire qui met en scène un train à grande vitesse Talgo longeant la plage. Cette image, publiée au moment où le premier AVE arrive à Gandía par des voies conventionnelles (grâce à la technologie des échangeurs d'écartement des essieux, certains trains peuvent circuler sur les deux réseaux) synthétise l'accessibilité et la modernité revendiquées par la ville. Pourtant, aucune desserte par la LGV n'est prévue pour cette station balnéaire dans les différents plans d'infrastructures. Plus qu'un moyen de transport, l'AVE est ici mis en scène comme un facteur de compression des distances et comme un support de développement touristique.

\section{B. Un changement radical de perspective}

La grande vitesse est un élément central du corridor méditerranéen en tant qu'elle cristallise les revendications et bénéficie d'une image forte. Toutefois, du point de vue de la rentabilité économique et de priorités affichées par la planification européenne, elle est souvent mise au second plan. Au regard de ce statut particulier de présence- 
absence endossé par la grande vitesse dans le corridor méditerranéen, la question du rôle de la grande vitesse dans la gouvernance s'éclaire d'un jour nouveau. En effet, l'on se demande habituellement qui sont les acteurs de la grande vitesse ferroviaire et quel type de gouvernance ils mettent en œuvre, mais le corridor méditerranéen invite à renverser cette perspective, car la grande vitesse est en réalité un acteur à part entière - plus qu'un facteur - de la gouvernance du corridor méditerranéen.

En effet, la grande vitesse est personnifiée, comme on l'a vu à travers les publicités et les discours : elle cristallise les objectifs d'acteurs aux profils très différents et elle crée un consensus entre les différentes échelles territoriales. En ce sens, tout en n'étant qu'une partie du projet de corridor méditerranéen et peut-être parce qu'elle en est la partie la plus soumise à caution, elle est un facteur très actif, et l'on peut même dire un acteur, de la recomposition des rapports de forces. Les grilles d'analyse traditionnelles de la gouvernance ne sont donc pas ici satisfaisantes.

Le corridor méditerranéen, qui tient un difficile équilibre entre grande vitesse et fret, et entre acteurs publics et privés, voit la grande vitesse prendre une part importante de l'espace médiatique parce qu'elle est investie d'un certain poids symbolique. Elle devient un acteur au sens où, portée en étendard par une échelle locale vindicative et soutenue comme opportunité de développement territorial, elle oblige l'ensemble des parties prenantes à une relecture du projet de corridor méditerranéen. Cette relecture se traduit d'une part par des modifications concrètes du projet (en matière de tracé, de choix des gares, de normes...) et d'autre part par des modifications progressives du sens donné au corridor méditerranéen comme objet politique. La grande vitesse participe à la négociation moins comme un objet des débats que comme un point de vue qui, s'il est lésé, exige compensation. Ainsi, c'est désormais moins pour obtenir une ligne à grande vitesse que l'on sait de plus en plus hypothétique que pour négocier des aménagements de ligne de passagers et des raccordements à une future ligne de fret que l'AVE est brandi sur l'axe méditerranéen. Une nouvelle approche de la gouvernance du corridor méditerranéen suppose de prendre en compte le décalage entre les poids symboliques respectifs de la grande vitesse et $d u$ fret - favorables à la première - et leurs poids réels dans les projets d'infrastructures - favorables au second.

Tel un coin dans le jeu des acteurs, le principe de subsidiarité modifie le poids de l'échelon national (très fort dans le gouvernement des infrastructures, mais affaibli dans la négociation sur le corridor méditerranéen), l'expression des régionalismes, facteur important de revendication, et enfin l'action des lobbies qui, non contente de porter le local et le régional à Bruxelles, introduit directement les acteurs privés dans l'organisation du débat politique. Ces nouveaux enjeux viennent bousculer la gouvernance traditionnelle et rendent nécessaire l'invention de nouvelles formes de rapport de forces qui, dans le cas du corridor méditerranéen, semblent se fédérer autour de la grande vitesse.

\section{Conclusion}

Il est fréquent de confondre le corridor méditerranéen avec le projet de ligne à grande vitesse qui en fait partie, car c'est celui-ci qui est le plus médiatisé. Néanmoins, il n'apparait que comme la scène sur laquelle, ou le prisme à travers lequel se posent les débats sur le corridor méditerranéen. En effet, la grande vitesse permet de donner corps au projet dans les territoires à l'échelle locale, tout en étant la partie la plus 
socialement acceptable du corridor: modernité, métropolisation à l'échelle méditerranéenne et européenne, aménagement du territoire et renouvellement urbain sont autant de thèmes relativement fédérateurs.

On est donc face à un grand écart du local à l'européen qui représente un saut qualitatif d'échelles, rendu possible par le principe de subsidiarité, et qui tient à l'écart un échelon national à la légitimité contestée par les régions méditerranéennes. En ce sens, la gouvernance du corridor méditerranéen est d'autant plus singulière qu'elle est porteuse d'une part des revendications régionalistes, voire indépendantistes. La grande vitesse, comme possible liaison forte entre Barcelone et Valence, comme raccordement direct des provinces littorales à l'Europe, est donc investie du poids de ces débats géopolitiques.

Une approche particulière de la gouvernance dans le corridor méditerranéen semble nécessaire. Il y a en effet beaucoup d'acteurs, mais c'est le dialogue entre les différentes facettes du projet qui est central. Il n'est en outre pas possible de séparer gouvernance de projet et gouvernance territoriale car le corridor méditerranéen, projet à long terme, a d'ores et déjà des implications territoriales fortes, qui l'ancrent dans le territoire avant que l'infrastructure ne soit réalisée. En découle un processus de gouvernance fait de l'intersection de plusieurs cadres usuels de la gouvernance de projet et de la gouvernance territoriale où la question de la grande vitesse cristallise les enjeux pour devenir un acteur à part entière de l'articulation entre les intérêts des différentes échelles territoriales.

\section{BIBLIOGRAPHY}

Abad García V., 1981, « Los primeros intentos de ordenación de la exportación citrícola: proyectos de Bellver y Doménech (1927-1928)», Saitabi, n³1, p.109-120.

Audikana A., 2012, La politisation de la grande vitesse espagnole (1986-2011): construction d'un mythe, production d'un consensus, émergence d'une controverse, Thèse de doctorat, Université Paris-Est, $423 \mathrm{p}$.

Bellet C., Alonso Logroño M.P., Casellas A., 2010, « Infraestructuras de transporte y territorio. Los efectos estructurantes de la llegada del tren de alta velocidad en España », Boletín de la Asociación de Geógrafos Españoles, n52, p.143-163.

Bellet C., Gutiérrez A., 2011, « Ciudad y ferrocarril en la España del siglo XXI. La integración de la alta velocidad en el medio urbano », Boletín de la Asociación de Geógrafos Españoles, n55, p.251- 279.

Bonerandi E., Santamaria F., 2011, « De la gouvernance à la gouvernance territoriale : enjeu de l'européanisation de l'aménagement du territoire. Réflexions à partir d'une recherche dans le cadre de l'Observatoire en réseau de l'aménagement du territoire européen (ORATE) », Cybergeo : European Journal of Geography (en ligne), Aménagement, Urbanisme, document 522, mis en ligne le 18 mars 2011, consulté le 11 juin 2014, URL : http://cybergeo.revues.org/23530 ; DOI : $10.4000 /$ cybergeo. 23530 
Camisón Zozorna C., 2012, Efectos del corredor mediterraneo en la competitividad de la economia valenciana, rapport, Asociación Valenciana de Empresas, 289 p.

CENIT, 2012, Mediterranean Corridor's south layout proposed by Ferrmed, Demonstrative Study, Barcelone, Ferrmed, $154 \mathrm{p}$.

Chevallier J., 2003, « La gouvernance, un nouveau paradigme étatique ? ", Revue française d'administration publique, Vol. 1, n¹05-106, p.203-217.

Commission européenne, 1996, Decision No 1692/96/EC of the European Parliament and of the Council of 23 July 1996 on Community guidelines for the development of the trans-European transport network, Décision de la Commission européenne, Bruxelles, 105 p.

Commission européenne, 2005, Trans-European Transport network: TEN-T priority axes and projects 2005, rapport de la Commission européenne, Luxembourg, 72 p.

Commission européenne, 2013, The Core Network Corridors. Trans European Transport Network 2013, rapport de la Commission européenne, Bruxelles, 48 p.

Debarbieux B., Vanier M., Beauchard J., 2002, Ces territorialités qui se dessinent, La Tour d'Aigues, éditions de l'Aube, $267 \mathrm{p}$.

Debrie J., Comtois C., 2010, « Une relecture du concept de corridors de transport: illustration comparée Europe/Amérique du Nord », Les Cahiers scientifiques du transport, n58, p.127-144.

Díaz Márquez S.E., Ureña Francés J.M., 2010, « El estudio del papel territorial de los intercambiadores de transporte: revisión y propuesta metodológica », Boletín de la Asociación de Geógrafos Españoles, n54, p.29-56.

Fabra M., 1975, « Valencianisme i Economía: Ignasi Villalonga (1895-1973) », Arguments, n², p.59184.

Ferrmed, 2009, Ferrmed global study. Ferrmed Great Axis Rail Freight Network and its area of influence. rapport pour la Commission européenne, Bruxelles, 205 p.

Institut Ignasi Villalonga, 2010, La Via economica de l'Euram. 10 anys, Valence (Espagne), Institut Ignasi Villalonga d'Economia i Empresa, $194 \mathrm{p}$.

Libourel E., 2011, L'insertion de Valence dans le corridor ferroviaire méditerranéen. Stratégies multiscalaires et aménagement urbain, Mémoire de Master 2, Champs-sur-Marne, Université ParisEst, $168 \mathrm{p}$.

MEDOCC, 2004, Le système métropolitain méditerranéen. Vers la création d'une zone d'intégration mondiale en Méditerranée, Livre blanc, Institut de la Méditerranée, 294 p.

Ministerio de Fomento, 2005, PEIT: Plan estratégico de infraestructuras y transporte : 2005-2020., Madrid, Ministerio de Fomento, 182 p.

Ministerio de Fomento, 2012, Plan de Infraestructuras, Transporte y Vivienda (2012-2024), Madrid, Ministerio de Fomento, $386 \mathrm{p}$.

MOPT, 1993, Plan Director de Infraestructuras 1993-2007, Madrid, MOPT, 379 p.

Offner J.-M., 1993, « Les "effets structurants du transport : mythe politique, mystification scientifique ", L’Espace géographique, Vol. 22, n³, p.233-242.

Prentice B.E., 1996, Transport gateways and trade corridors, University of Saskatchewan Printing Services, Saskatoon. 
Priemus H., Zonneveld W., 2003, « What are corridors and what are the issues? Introduction to special issue: the governance of corridors ", Journal of Transport Geography, Vol. 11, n³, p.167-177.

Rey-Valette H., Chia E., Mathé S., Michel L., Nougarèdes B., Soulard C.-T., Maurel P., Jarrige F., Barbe E., Guiheneuf P.-Y., 2014, « Comment analyser la gouvernance territoriale ? Mise à l'épreuve d'une grille de lecture ", Géographie, économie, société, Vol. 16, 1, p.65-89.

Romero González J., 2005, « El gobierno del territorio en España. Balance de iniciativas de coordinación y cooperación territorial », Boletín de la Asociación de Geógrafos Españoles, n³9, p.5986.

Romero González J., 2012, « España inacabada. Organización territorial del Estado, autonomía política y reconocimiento de la diversidad nacional ", Documents d'anàlisi geogràfica, n 58, p.13 - 49. Romero González J., Farinós Dasí J., 2012, « Cities and Urban and Metropolitan Regions in Spain: A New Agenda in a Global Context », in João Seixas and Abel Albet, Urban Governance in Southern Europe, Farnham, Ashgate, p.123-148.

Santos y Ganges L., 2007, Urbanismo y ferrocarril: la construcción del espacio ferroviario en las ciudades medias españolas, Madrid, Fundación de los Ferrocarriles Españoles, 460 p.

Trépier C., 2013, « L'indépendance de la Catalogne, un débat européen d'abord politique », L’Espace Politique. Revue en ligne de géographie politique et de géopolitique (en ligne), Vol. 21, n³, mis en ligne le 19 novembre 2013, consulté le 05 juin 2014. URL : http://espacepolitique.revues.org/ 2828

Vanier, M. (dir.), 2009, Territoires, territorialité, territorialisation: controverses et perspectives, Rennes, Presses universitaires de Rennes, $228 \mathrm{p}$.

Whebell C.F.J., 1969, « Corridors: A Theory of Urban Systems », Annals of the Association of American Geographers, Vol. 59, n¹, p.1-26.

Woessner R., 2010, « La territorialisation : proposition pour la compréhension du phénomène par une entrée systémique », Revue d'Économie Régionale \& Urbaine, octobre, n4, p. 669.

\section{ABSTRACTS}

In the twofold context of the development of Trans-European Networks of Transport (TEN-T) and the construction of a high-speed network in Spain, the Mediterranean railway corridor (MRC) appears as a powerful element of territorialisation. This continually developing object causes strong, modifications in the governance processes, transforming power relations between public and private stakeholders at all scales and challenging the current institutional decision-making processes. Moreover, even though MRC should host freight and passengers, the project of highspeed deployment on the Mediterranean coast is of particular importance. This has a positive image and consequently involves many local stakeholders. Such a project would be the very first major passenger axis in Spain that does not cross Madrid, which makes it a geopolitical object. We will therefore try to highlight the way new actors have emerged within the MRC project and investigate their positioning in this particular contentious context of multiscalar governance.

Dans le double contexte des Réseaux transeuropéens de Transport (RTE-T) et de la constitution d'un réseau à grande vitesse en Espagne, le corridor ferroviaire méditerranéen apparaît comme un élément puissant de territorialisation. Ce projet, qui connaît des développements permanents, contribue à provoquer des changements majeurs dans les processus de gouvernance. Il modifie en effet les rapports de force entre acteurs privés et publics à toutes les échelles, et il interroge 
les processus habituels de prise de décision. De plus, quoique le corridor ferroviaire méditerranéen soit à la fois un axe de fret et de voyageurs, le projet de développement de la grande vitesse le long du littoral méditerranéen revêt une importance toute particulière, grâce à son image jugée positive qui implique de nombreux acteurs locaux. Il s'agirait en effet du premier axe de passagers important ne passant pas par Madrid, ce qui en fait un objet géopolitique. C'est pourquoi nous proposons de montrer comment de nouveaux acteurs ont émergé autour du projet de corridor méditerranéen et de décrire la manière dont ils prennent position dans ce contexte crispé de gouvernance multiscalaire.

Im doppelten Rahmen von der Entwicklung der Transeuropäischen Verkehrsnetze und der Einsetzung eines Hochgeschwindigkeitsnetzes in Spanien lässt sich das Mittelmeerseisenbahnkorridor als wichtige Grundlage der Territorialisierung. Dieses ständig wandelnde Konzept trägt zu wesentlichen Änderungen der Governanceprozesse bei. Dadurch werden Machtverhältnisse zwischen privaten und öffentlichen Akteuren auf alle Ebene verändert und traditionelle Entscheidungsprozesse in Frage gestellt. Obwohl dieser Eisenbahnkorridor gleichzeit Fracht und Zugfahrgäste gewidmet wird, ist die Entwicklung von Hochgeschwindigkeit an der Mittelmeerküste entlang, da sein positives Image viele lokale Akteuren mit sich bringt, von hoher Bedeutung. Er ware die allererste wesentliche Zugverkehrsachse für Fahrgäste, die nicht über Madrid fahren würde. Dadurch wird er einen geopolitischen Gegenstand. In dieser Hinsicht wollen wir zeigen, wie neue Akteure im Rahmen dieses Projekts aufgetaucht sind und wie sie sich in einem gespannten Kontext von einer mehrstufigen Governance gegeneinander positionieren.

\section{INDEX}

Mots-clés: acteurs, corridor méditerranéen, Espagne, RTE-T

Schlüsselwörter: Akteuren, Mittelmeerseisenbahnkorridor, Spanien, Transeuropäischen Verkehrsnetze

Keywords: Mediterranean corridor, Spain, stakeholders, TEN-T

\section{AUTHOR}

\section{ELOÏSE LIBOUREL}

Université Paris-Est - Laboratoire Ville Mobilité Transport (ENPC/UPEM/IFSTTAR) - UMR-T 9403

- Bâtiment Bienvenüe - 6-8 avenue Blaise Pascal Champs-sur-Marne 77455 Marne-la-Vallée Cedex 2 - eloise.libourel@enpc.fr 